\title{
Práticas da família sobre cuidados em saúde frente às doenças respiratórias infantis durante as queimadas
}

\author{
Family practices on health care facing children's respiratory diseases during burns
}

\author{
Prácticas familiares sobre el cuidado de la salud frente a las enfermedades respiratorias \\ del niño durante las quemaduras
}

Isabele Cristina Mascarenhas Costa ${ }^{1 *}$, Maria Michelle Pereira dos Santos ${ }^{1}$, Marcela Milrea Araújo Barros $^{1}$,Gabriela Aparecida Paz de Castro Barreto ${ }^{1}$,Maria de Fátima Cândida da Silva ${ }^{1}$,Thainá França Oliveira ${ }^{1}$,Gisele da Silva Cardoso Fernandes ${ }^{1}$, Thaís Millena Cardoso de Lima ${ }^{1}$.

\begin{abstract}
RESUMO
Objetivo: Conhecer as práticas de prevenção e cuidados relacionados às doenças respiratórias no período das queimadas florestais na perspectiva dos genitores/cuidadores de crianças de 29 dias e 12 anos em uma unidade básica de saúde da Amazônia Legal. Métodos: Trata-se de uma pesquisa qualitativa, exploratória e descritiva com uso da técnica entrevista com roteiro semiestruturado em um município da Região Norte do Brasil. Resultados: A amostra desta pesquisa foi constituída por 20 mulheres, das quais 19 mães e 1 avó. As participantes, referiram dificuldades para identificar sintomas de gravidade na criança relacionados as doenças respiratórias. Durante o período de queimadas florestais, mencionaram a tosse e a dificuldade para respirar como sintomas relacionados à exposição da criança à fumaça. A automedicação é aplicada como estratégia frente às barreiras de acesso à atenção primária como dificuldades para agendar consultas e longa espera para atendimento, nessas condições, recorrem ao serviço de urgência, contribuindo para superlotação e internação. Conclusão: Existe a necessidade de uma reorganização nas redes de atenção à saúde com um olhar para o binômio família/criança como um grupo de vulnerabilidade nas queimadas sazonais e a implementação de estratégias de educação em saúde pelo enfermeiro.
\end{abstract}

Palavras-chave: Doenças respiratórias, Família, Criança.

\begin{abstract}
Objective: To know the prevention and care practices related to respiratory diseases during the period of forest fires from the perspective of parents/caregivers of children aged 29 days and 12 years in a basic health unit in the Legal Amazon. Methods: This is a qualitative, exploratory and descriptive research using the interview technique with a semi-structured script in a municipality in the North of Brazil. Results: The sample of this research consisted of 20 women, including 19 mothers and 1 grandmother. Participants reported difficulties in identifying serious symptoms in children related to respiratory diseases. During the period of forest fires, they mentioned coughing and difficulty breathing as symptoms related to the child's exposure to smoke. Self-medication is applied as a strategy to face barriers to accessing primary care, such as difficulties in scheduling appointments and long waits for care, under these conditions, they resort to the emergency service, contributing to overcrowding and hospitalization. Conclusion: There is a need for a reorganization in health care networks with a view to the family/child binomial as a vulnerable group in seasonal fires and the implementation of health education strategies by nurses.
\end{abstract}

Keywords: Respiratory diseases, Family, Child.

${ }^{1}$ Faculdade Interamericana de Porto Velho - UNIRON, Porto Velho - RO.

*E-mail: Isabele.cristina321@gmail.com

SUBMETIDO EM: 6/2021

ACEITO EM: 6/2021

PUBLICADO EM: 6/2021 


\section{RESUMEN}

Objetivo: Conocer las prácticas de prevención y atención relacionadas con las enfermedades respiratorias durante el período de incendios forestales desde la perspectiva de los padres / cuidadores de niños de 29 días y 12 años en una unidad básica de salud en la Amazonía Legal. Métodos: Se trata de una investigación cualitativa, exploratoria y descriptiva mediante la técnica de entrevista con guion semiestructurado en un municipio del norte de Brasil. Resultados: La muestra de esta investigación estuvo constituida por 20 mujeres, incluidas 19 madres y 1 abuela. Los participantes informaron dificultades para identificar síntomas graves en niños relacionados con enfermedades respiratorias. Durante el período de los incendios forestales, mencionaron tos y dificultad para respirar como síntomas relacionados con la exposición del niño al humo. La automedicación se aplica como estrategia para enfrentar barreras de acceso a la atención primaria, como dificultades en la programación de citas y largas esperas de atención, en estas condiciones recurren al servicio de emergencia, contribuyendo al hacinamiento y la hospitalización. Conclusión: Existe la necesidad de una reorganización en las redes de atención de salud con miras al binomio familia / niño como grupo vulnerable en incendios estacionales y la implementación de estrategias de educación en salud por parte de enfermeras.

Palabras clave: Enfermedades respiratorias, Familia, Niño.

\section{INTRODUÇÃO}

As doenças do trato respiratório podem estar relacionadas pela exposição à poluição atmosférica emitida em grande escala em todo o planeta. Existe uma associação significativa entre os níveis desses poluentes e o número de atendimentos em emergências e internações por causas respiratórias (MATOS EP, et al., 2019).

As queimadas, na maior parte dos casos, são oriundas das ações humanas. Por encontrarem condições propícias, na estação de estiagem ou seca, principalmente nas regiões da Amazônia Legal, os incêndios se propagam em alta magnitude, pois os fatores climáticos e meteorológicos, tais como altas temperaturas, baixa umidade do ar e vento, potencializam a sua ocorrência, e ocasionam maior atuação da radiação solar sobre a superfície terrestre. Esta por sua vez, elevam a temperatura do ar, criando um ambiente suscetível ao processo de queima, ocasionando danos respiratórios à vida humana (VIGANO HHG, et al., 2018).

No mundo, $93 \%$ das crianças vivem em ambientes onde as concentrações de gases poluentes estão acima das preconizadas pela Organização Mundial de Saúde (OMS). Estima-se que uma em cada quatro mortes de crianças menores de 5 (cinco) anos tenha relação direta ou indireta aos riscos ambientais (SANTOS UP, et al., 2021).

No Brasil em torno de 421 mil internações de crianças entre 0 e 10 anos de idade são decorrentes de doenças respiratórias, ocasionando custos de $R \$ 85$ milhões ao Sistema Único de Saúde (SUS). Estudos epidemiológicos têm avaliado os impactos de poluentes do ar sobre a saúde da população incluindo taxas de mortalidade, de internação e atendimentos emergenciais por doenças respiratórias (MACHIN $A B$ e NASCIMENTO LFC 2018).

No Estado de Rondônia (RO), cenário de investigação deste estudo, entre os anos de 2012 a 2016, foram analisadas 133.958 notificações de serviços públicos e conveniados com o Sistema Único de Saúde (SUS) nos 52 municípios. Destes, $24,8 \%$ dos pacientes necessitaram de hospitalização dos quais $14,8 \%$ eram crianças menores de cinco anos (FREITAS JLG, et al., 2020).

Nos cuidados à saúde da população, a atenção à criança representa um campo prioritário, considerando sua fragilidade e vulnerabilidade ao adoecimento e sua consequente gravidade em função das características próprias da idade (PEDRAZA DF e ARAUJO EMN, 2017).

A família neste cenário é de suma importância para o desenvolvimento de cuidados à criança. Em casos de doenças respiratórias, os cuidadores podem atuar juntamente com a equipe de enfermagem na promoção à saúde, considerando que a presença da doença aguda ou crônica, interfere diretamente no cotidiano das crianças e de suas famílias (SILVEIRA A e NEVES ET, 2016). 
O Ministério da Saúde (MS) estabeleceu em 2015, a Política Nacional de Atenção Integral à Saúde da Criança (PNAISC) com a Portaria ํㅜ 1.130, que se organiza a partir das redes de atenção à saúde, a qual sintetiza de modo claro e objetivo os eixos de ações que associam a atenção integral à saúde da criança visando estratégias e dispositivos que contribuam para a implementação dessas ações nos serviços de saúde pela gestão municipal e estadual, bem como, pelos profissionais de saúde (DAMASCENO SS, et al., 2016).

Sem uma assistência oportuna pela Atenção Primária à Saúde (APS), há uma predominância maior de doenças respiratórias, estas, são denominadas Internações por Condições Sensíveis à Atenção Primária (ICSAP). Construída com base na Décima Revisão Internacional de Doenças (CID-10), as ICSAP são indicadores de morbimortalidade que tomam como base, problemas de saúde para os quais a APS pode minimizar o risco de internações (PEDRAZA DF e ARAUJO EMN, 2017).

Diante disso, a APS distingue-se por promover maior eficácia no fluxo dos usuários dentro do sistema de saúde, tratamento mais efetivo de condições crônicas, maior efetividade do cuidado, máxima utilização de técnicas preventivas, maior satisfação dos usuários e redução das iniquidades (NEVES ET, et al., 2019).

Os cuidadores na maioria das vezes são os primeiros a identificar os sinais e sintomas de uma infecção respiratória aguda em crianças (PASSOS SD, et al., 2018). Diante disso, a questão que norteará este estudo é: quais os cuidados praticados pela família frente às doenças respiratórias infantis no período das queimadas sazonais no Norte do Brasil?

Esta pesquisa objetiva conhecer as práticas de prevenção e cuidados relacionados às doenças respiratórias no período das queimadas florestais na perspectiva dos genitores/cuidadores de crianças de 29 dias a 12 anos em uma unidade básica de saúde (UBS) da Amazônia Legal.

\section{MÉTODOS}

Trata-se de uma pesquisa de campo de abordagem qualitativa e descritiva, realizada com genitores ou cuidadores de crianças atendidas em uma UBS da Amazônia Legal localizada na região Norte do Brasil. Utilizamos como instrumento de coleta, um roteiro semiestruturado com uso da técnica entrevista.

A população foi constituída por mães/cuidadoras de crianças com idade entre 29 dias e 12 anos, atendidas nos programas da Unidade Básica de Saúde (UBS) e que aceitaram participar voluntariamente da pesquisa. A escolha desta população foi baseada na Lei $n^{\circ} 8.069$, de 13 de julho de 1990 que considera criança, a pessoa com idade até 12 (doze) anos incompletos (11 meses e 29 dias de idade).

Como critério de seleção amostral, utilizou-se o tipo não probabilístico, por conveniência, dos quais foram convidados um $n^{\circ}$ de 20 participantes que concordaram ingressar voluntariamente no estudo, considerando os critérios de inclusão.

Todos os praticantes desta pesquisa receberam e assinaram por livre arbítrio o Termo de Consentimento Livre e Esclarecido (TCLE). A pesquisa obteve o Certificado de Apresentação de Apreciação Ética (CAAE) com o n. ${ }^{-}$32906920.3.0000.8028 e foi aprovada no mês de agosto do ano de 2020 sob o parecer no 4.191 .431 , pelo Comitê de Ética em Pesquisa (CEP) da União Educacional do Norte Ltda (UNINORTE) em consideração aos pressupostos contidos na Resolução 466/12 do Conselho Nacional de Saúde/ Ministério da Saúde, que aprova as diretrizes e normas regulamentadoras de pesquisas envolvendo seres humanos.

Os resultados das entrevistas foram analisados mediante análise de conteúdo. Após a gravação e transcrição das entrevistas na íntegra, elas foram destruídas para manter o sigilo das informações, posteriormente analisadas de acordo com a literatura pertinente.

Vale ressaltar que, levando em consideração que este estudo foi realizado durante a pandemia da Covid19, medidas de segurança como, o uso de Equipamentos de Proteção Individuais (EPIs), antissepsia das mãos com álcool em gel, canetas higienizadas a cada troca de entrevistado, ambiente arejado, privativo e livre de aglomerações, foram adotadas pelas pesquisadoras durante a coleta para garantir e preservar a saúde de todos os envolvidos nesta pesquisa. 


\section{RESULTADOS E DISCUSSÃO}

A amostra desta pesquisa foi constituída por 20 mulheres, sendo 19 mães e 1 avó de crianças com idade entre 29 dias a 12 anos atendidas nos programas da UBS cenário do estudo. Não houveram cuidadores genitores do sexo masculino. Duas pesquisadas encontravam-se gestantes no dia da coleta, uma a espera do quarto filho e a outra aguardava a chegada do quinto. Nove participantes possuem somente 1 filho, seis têm 3 filhos e três são mães de 2 filhos. No que se refere à escolaridade das cuidadoras, cinco não concluíram o ensino fundamental, apenas uma conseguiu concluir, quatro não terminaram o ensino médio e sete possuem ensino médio completo, somente duas possuem o ensino superior completo e uma o ensino superior incompleto (Quadro 1).

Quadro 1 - Perfil das participantes da pesquisa.

\begin{tabular}{|c|c|c|c|}
\hline $\begin{array}{c}\text { Código de } \\
\text { identificação }\end{array}$ & $\begin{array}{c}\text { Grau de } \\
\text { parentesco }\end{array}$ & No de filhos & Escolaridade \\
\hline P1 & Mãe & 1 & Ensino Médio incompleto \\
\hline P2 & Mãe & 1 & Ensino Superior completo \\
\hline P3 & Mãe & 1 & Ensino Superior completo \\
\hline P4 & Mãe & 3 & Ensino Médio completo \\
\hline P5 & Mãe & 3 & Ensino Médio completo \\
\hline P6 & Mãe & 3 & Ensino Fundamental incompleto \\
\hline P7 & Mãe & 1 & Ensino Médio completo \\
\hline P8 & Mãe & 3 & Ensino Fundamental completo \\
\hline P9 & Mãe & 4 & Ensino Fundamental incompleto \\
\hline P10 & Avó & 1 & Ensino Fundamental incompleto \\
\hline P11 & Mãe & $3^{*}$ & Ensino Médio incompleto \\
\hline P12 & Mãe & 2 & Ensino Médio completo \\
\hline P13 & Mãe & 2 & Ensino Médio incompleto \\
\hline P14 & Mãe & 1 & Ensino Fundamental incompleto \\
\hline P15 & Mãe & 1 & Ensino Médio completo \\
\hline P16 & Mãe & $4^{* *}$ & Ensino Fundamental incompleto \\
\hline P17 & Mãe & 3 & Ensino Médio completo \\
\hline P18 & Mãe & 1 & Ensino Superior Incompleto \\
\hline P19 & Mãe & 2 & Ensino Médio incompleto \\
\hline P20 & Mãe & 1 & Ensino Médio completo \\
\hline
\end{tabular}

Legenda: *Gestante do quarto filho; ${ }^{*}$ Gestante do quinto filho.

Fonte: Costa ICM, et al., 2021.

A partir da análise de dados, emergiram três núcleos temáticos: "Cuidados realizados pela família diante dos sintomas respiratórios no período das queimadas", "Dificuldades de acesso aos serviços de saúde à criança" e "Contribuições sugeridas para minimizar as condições respiratórias infantis no período das queimadas". Essas temáticas estão baseadas nos relatos semelhantes e condizentes contidos na matriz de síntese.

\section{Cuidados realizados pela família diante dos sintomas respiratórios no período das queimadas}

Para o crescimento e desenvolvimento de crianças, a qualidade do cuidado é um fator fundamental e depende, em primeiro lugar, de condições psicossociais, sanitárias e econômicas adequadas. A deficiência no meio ambiente em que a criança convive, pode promover riscos ao desenvolvimento infantil (FREITAS BHBM, et al., 2021). 
Em referência a piora dos sintomas na criança em época de queimadas relacionados a fumaça, as participantes familiares cuidadoras referem que:

“(...) na época da queimada ele fica com dificuldade de respirar, dá tosse nele e o nariz fica entupido" (P1).

“(...) ela começa a tossir muito, tosse bastante quando começa esses tempos de queimadas" (P8).

As crianças são mais vulneráveis a fatores externos, como a poluição, pois seu sistema imunológico ainda está em formação e o aparelho respiratório, em desenvolvimento. Além disso, crianças passam mais tempo ao ar livre do que os adultos e, assim, inalam mais gases poluentes (BRASIL, 2019).

Como situação que deve gerar inquietação e alerta por parte dos profissionais de saúde e gestores, as falas abaixo referem que as mães/cuidadoras apresentam dificuldades para identificar sintomas de gravidade de doenças respiratórias:

"Na realidade não sei identificar quais são os sintomas" (P6).

"Ainda não, eu queria aprender a conhecer esses sintomas, mas infelizmente eu ainda não sei (...)" (P14).

A falta de compreensão das participantes em relação a identificação dos sinais e sintomas e a necessidade de monitoramento da criança com suspeita de doença respiratória tem ocasionado à busca de terapêuticas inadequadas e, na maioria dos casos, essas cuidadoras procuram atendimento médico após o avanço no agravo da condição da criança, exigindo intervenção de resgate e tratamentos invasivos que em algumas situações podem não ser mais efetivos (PASSOS SD, et al., 2018).

São referidos como principais sintomas de doenças respiratórias a tosse, dificuldade para respirar, chiado no peito, dor de ouvido, dor de garganta e coriza (GONZALES YP, 2018). A maioria das tosses são causadas por infecções virais agudas em crianças saudáveis que podem anualmente se repetir de seis a dez vezes (NASCIMENTO WMC, 2018). Em complemento, a tosse na criança foi um dos sintomas mais referidos pelas cuidadoras:

"(...) é tosse, as vezes um pouquinho de falta de ar, febre, muita coriza e mal-estar mesmo, moleza no corpo, até onde eu sei” (P7).

\section{“(...) é mais fácil identificar a tosse (...)" (P20).}

A dificuldade de respirar é outro sintoma evidente no discurso de alguns participantes desta pesquisa e, segundo Passos SD, et al. (2018), a dispneia é um sinal de alerta precoce e precede as alterações nos gases no sangue. Portanto, identificar esse sintoma em crianças é fundamental para a detecção e tratamento precoce das doenças respiratórias, a fim de evitar complicações, tratamentos mais invasivos e até a morte.

"Ela fica com dificuldades de respirar (...)" (P3).

"Geralmente eles ficam com a respiração cansada..." (P4).

$\mathrm{Na}$ tentativa de tratar os sintomas respiratórios e pelas barreiras de acesso aos serviços de saúde, as cuidadoras frequentemente administram medicamentos livres de prescrição. Entretanto a eficácia destes medicamentos para tratar o resfriado em crianças, em sua maioria, não está comprovada. Além disso, o uso desse tipo de medicação em crianças está associado a erros de ingestão de doses tóxicas e efeitos adversos, levando a atendimentos de urgências e até a morte (NASCIMENTO WMC, 2018).

\section{Dificuldades de acesso aos serviços de saúde à criança}

Neves RG, et al. (2017), referem que em decorrência da dificuldade de acesso e baixa resolutividade dos serviços da Atenção Primária à Saúde (APS), as famílias da criança geralmente utilizam o pronto atendimento e pronto-socorro como principal escolha para resolução de problemas de saúde de baixa e média complexidade. 
Questionadas a respeito do acesso ao atendimento na UBS quando sua criança apresenta sintomas respiratórios no período sazonal de queima e fumaça, as participantes relatam barreiras de acesso à APS como porta de entrada para o cuidado, dificuldades no agendamento de consultas, longa espera por atendimento e até a insatisfatória receptividade dos profissionais.

“(...) você vem no posto e não tem médico ou tem que marcar e voltar tal dia, a gente acaba levando no particular ou então no pronto socorro mesmo porque se ele tiver muito ruim não tem como esperar não" (P7).

"Encontro dificuldades sim, com relação a unidade básica de saúde, as vezes eu encontro, aonde que eu vou mais? e que é mais rápido é lá no hospital infantil (...)" (P8).

“(...) tem muita gente que recebe a gente bem, mas tem muitos profissionais que não recebem. Demora bastante" (P9).

Segundo Rossato K, (2017), o acolhimento nos serviços de saúde deve estar presente no atendimento prestado aos usuários pelos profissionais de saúde, de forma a atender a todos, ouvindo suas solicitações e adotando uma atitude capaz de dar respostas adequadas, utilizando todos os meios disponíveis para a resolução dos problemas.

Outro fato que chama a atenção, além da dificuldade de acesso elencada pelas participantes desta pesquisa, é que, por não conseguirem o atendimento na UBS as mães/cuidadoras recorrem aos serviços de urgência e emergência e até mesmo os serviços privados, contribuindo para superlotação e em algumas situações hospitalizações.

"É difícil! Você vem no posto e não tem médico ou tu tens que marcar e voltar tal dia a gente acaba levando no particular ou então no pronto socorro mesmo porque se ele tiver muito ruim não tem como esperar não" (P7).

Dentro do serviço de pronto atendimento infantil, salienta-se a superlotação, isso faz com que prejudique a qualidade da assistência, pois para que os pacientes não sofram longa espera no atendimento juntamente aqueles que necessitam de orientações e cuidados primário é necessária agilidade no atendimento e, por sua vez, ocasionar comprometimento na eficácia do acolhimento (MACEDO GPOS, D'INNOCENZO M, 2019).

Alguns participantes da pesquisa relatam que somente buscam atendimento médico diante da não resolutividade dos sintomas através dos cuidados que realizam no domicílio.

$$
\begin{aligned}
& \text { “(...) só levo no posto de saúde em último caso (...)” (P4). } \\
& \text { “(...) e aí se piorar levo no médico” (P7). }
\end{aligned}
$$

Notadamente nos períodos de sazonalidade viral respiratória, a lotação nas emergências pediátricas passou a sobrecarregar os serviços, com prejuízo ao atendimento dos casos graves ou críticos e o desgaste das longas horas de espera daqueles com menor gravidade (SOUZA RR, et al., 2019).

Nesse contexto, a UBS acaba não satisfazendo as necessidades da população infantil, pois a família ainda não tem o entendimento sobre os serviços básicos como a promoção de saúde e prevenção de agravos e o acolhimento à demanda espontânea como de responsabilidade da APS. Assim, o fortalecimento da atenção básica, reduziria a demandas das unidades de urgência e emergência, promovendo a minimização do fluxo de atendimentos e internações hospitalares (BANDO DH e MOREIRA DG, 2019).

\section{Contribuições sugeridas para minimizar as condições respiratórias infantis no período das queimadas}

Com o objetivo de melhorar o controle das doenças respiratórias e minimizar complicações, a população necessita compreender a importância de práticas de cuidados apropriados e oportunos. Estratégias educativas, podem ser realizadas em grupos ou individualmente, encontrar formas de integrar diferentes mensagens educacionais é um desafio constante pois a educação eficaz do usuário ou sua família, envolve mudanças de comportamento que podem levar tempo (GONZALES YP, 2018). 
Na fala de uma das participantes, é possível perceber a deficiência de programas de educação em saúde nos serviços da APS que visem minimizar as complicações de doenças respiratórias na infância.

“(...) uma atenção especial com as crianças...para ter aquele acompanhamento mais de perto para saber mais ou menos se a criança já é propícia a ter problemas respiratórios, ter um cuidadinho a mais, porque tudo é na prevenção" (P4).

Vale ressaltar que, uma das características da APS é a orientação à família, analisando e compreendendo o processo saúde-doença no contexto familiar e da rede social por meio do estabelecimento de vínculos e do conhecimento da história das famílias e dos usuários com necessidades de saúde. A partir desse olhar, evidencia-se como uma estratégia oportuna uma abordagem integral à população infantil na Estratégia Saúde da Família (ESF) (BRITO GEG, et al., 2018).

Nota-se no relato de uma das mães, a importância da informação sobre os serviços e atribuições da APS, ao referir o desejo de um atendimento na UBS para facilitar o acesso à saúde da sua criança.

"Que a gente tivesse o atendimento aqui no postinho também, isso facilitaria bastante porque na Unidade de Pronto Atendimento (UPA) tem várias outras coisas e eles não dão muita atenção" (P19).

Um dos principais motivos de demanda por consultas são as doenças respiratórias, sendo assim, a base para alcançar os objetivos precisos é o trabalho em equipe organizado e planejado, incentivando a participação da comunidade na busca de soluções para os problemas de saúde (GONZALES YP, 2018).

É perceptível em alguns trechos de fala das participantes, a consciência ingênua em relação às queimadas florestais e sua proporção, com a citação por exemplo da queimada cultural do lixo doméstico. Entretanto, a maioria das entrevistadas expressaram o mesmo desejo para que não se pratiquem queimadas como sugestão para minimização dos casos de doenças respiratórias infantis.

"Que não queimassem, que não fizessem essas queimadas, mas fazer o que? A gente não pode mandar nesse pessoal que queima por aí, esses madeireiros, é a sugestão que eu dou (...)" (P7).

"Parar de estar queimando, eu acho que diminuiria bastante, porque as pessoas fazem muitas queimadas, ontem mesmo perto da minha casa tinha aquelas fumaças pretas subindo" (P8).

"Ah, é a população não tocar fogo no lixo doméstico (...)" (P9).

No mês de agosto de 2019, três milhões de pessoas moradoras da região amazônica foram expostas a poluentes que ultrapassaram o limite preconizado pela Organização Mundial da Saúde (OMS). No nono mês do mesmo ano, o número aumentou para quatro milhões e meio de pessoas expostas. Esses poluentes estão relacionados à ocorrência de queimadas na Amazônia e têm sido associados com as doenças respiratórias principalmente em grupos de vulnerabilidade. Essa exposição teve um aumento significativo a partir do mês de junho do ano de 2020 , onde se registrou quase $20 \%$ a mais de focos de calor em relação a junho de 2019 (ALENCAR A, et al., 2020).

Abaixo, uma mãe expressa sua inquietação ao referir que é difícil respirar pois em todos os lugares têm queimadas e a fumaça é visível.

"(...) todo lugar tem queimada e fica bem difícil, onde vai tem queimada aí fica difícil respirar desse jeito" (P20).

Como estratégia de gestão, o Ministério do Meio Ambiente (MMA), estabeleceu a Portaria de № 78, de 3 de março de 2021, a declaração do estado de emergência ambiental entre os meses de abril a novembro de 2021 no qual o Estado de Rondônia está inserido, justificado pelo período de estiagem das chuvas e aumento das queimadas florestais (BRASIL, 2021). Filho GD e Pires VO (2020), referem que a melhor estratégia para diminuir a degradação do meio ambiente é ampliar a educação ambiental, tornando-a de fato efetiva quando há a integração saúde e ambiente, com corresponsabilidade da população aos problemas ambientais e suas implicações na saúde e busquem a preservação e a melhoria do meio ambiente. 
É imprescindível, ainda, qualificar e reorganizar a rede de atenção à saúde com um olhar diferenciado para a criança e sua família, investindo na qualificação dos serviços e profissionais da APS para identificar e tratar adequadamente agravos evitáveis. Nesse cenário, a educação em saúde, deve abordar os cuidados necessários para a implementação de cuidados à criança pela família a fim de minimizar os riscos respiratórios e internações evitáveis.

\section{CONCLUSÃO}

As ações que envolvem a família da criança no contexto da APS ainda são limitadas nas abordagens da literatura científica. A família precisa estar inserida nesse processo em sua totalidade uma vez que, as práticas de cuidados realizadas frente às doenças respiratórias na criança em período de queimadas sazonais, devem ser estratégia de atuação da atenção básica, para que juntas proporcionem uma assistência que minimizem a morbimortalidade infantil. Neste contexto, o profissional enfermeiro também tem um importante papel no desenvolvimento de ações na APS, além de realizar educação em saúde, promove o acolhimento e bemestar dos usuários de forma humanizada e qualificada com base em evidências científicas.

\section{REFERÊNCIAS}

1. ALENCAR A, et al. O fogo e o desmatamento em 2019 e o que vem em 2020; O Instituto de Pesquisa Ambiental da Amazônia (IPAM) em 29 de abril den. ํㅜㄹ, pp. 2020.

2. BANDO DH, MOREIRADG. Territorialização Da Atenção Básica À Saúde Do Sus No Município De Alfenas (Mg): Estudo De Caso Sobre A UBS Jardim Nova América; Geo saúde- 2019;

3. BRASIL, Diário Oficial da União o Órgão: Ministério do Meio Ambiente/Gabinete do Ministro; PORTARIA MMA № 78, DE 3 DE MARÇO DE 2021; 2021; 43(1): 127.

4. BRASIL. Fundação Oswaldo Cruz (Fiocruz) - Agência de Notícias; Queimadas afetam saúde de crianças na Amazônia; Rev. Colabora, 2019; 18-47;

5. BRITO GEG, et al. O objeto de trabalho na Estratégia Saúde da Família. Interface (Botucatu), Botucatu, 2018; 22(64): 77- 86 .

6. DAMASCENO SS, et al. Saúde da criança no Brasil: orientação da rede básica à Atenção Primária à Saúde. Ciênc. Saúde coletiva, Rio de Janeiro, 2016; 21(9): 2961-2973.

7. FILHO GD, PIRES VO. Práticas Educacionais De Controle das Queimadas Para Preservação Do Meio Ambiente No Município De Barra Do Garças-MT; Rev. Interfaces do Conhecimento 2020; 2(3).

8. FREITAS BHBM, et al. O trabalho emocional em enfermagem pediátrica face às repercussões da COVID-19 na infância e adolescência. Rev. Gaúcha Enferm., Porto Alegre, 2021; 42: e20200217.

9. FREITAS JLG, et al. Internações por condições sensíveis à atenção primária em crianças em Rondônia de 2008 a 2017. Cogitareenferm. Apa; 2020; 25: e71904.

10. GONZALESYP, Estratégia educativa para diminuir as Infecções Respiratórias Agudas na comunidade de Adrianópolis, Paraná; Universidade Federal de Santa Catarina (UFSC); 2018.

11. MACEDO GPOS, D'INNOCENZO M. A satisfação dos familiares sobre o fluxo de atendimento no Pronto-Socorro Pediátrico. Rev. Bras. Enferm., Brasília, 2019; 72(2): 435-441.

12. MACHIN AB; NASCIMENTO LFC. Efeitos da exposição a poluentes do ar na saúde das crianças de Cuiabá, Mato Grosso, Brasil. Cad. Saúde Pública, Rio de Janeiro,2018; 34(3): e00006617.

13. MATOS EP, et al. Análise espaço-temporal do efeito da poluição do ar na saúde de crianças. Cad. Saúde Pública, Rio de Janeiro, 2019; 35(10): e00145418.

14. NASCIMENTO WMC. Xarope de chambá (justiciapectoralisjacq.) No tratamento da tosse e sintomas respiratórios: um ensaio clínico randomizado. 2018pp. $73 \mathrm{f}$.

15. NEVES ET, et al. Acesso de crianças com necessidades especiais de saúde à rede de atenção. Rev. Bras. Enferm., Brasília, 2019; 72(3): 65-71.

16. NEVES RG, et al. Cobertura da vacina Meningocócica C nos estados e regiões do Brasil em 2012. Revista Brasileira de Medicina de Família e Comunidade, 2016;11(38): 1-10.

17. PASSOS SD, et al. Doenças Respiratórias Agudas em Crianças Brasileiras: os cuidadores são capazes de detectar os primeiros sinais de alerta? Rev. paul. pediatr., São Paulo, 2018; 36(1): 3-9.

18. PEDRAZA DF, ARAUJO EMN. Internações das crianças brasileiras menores de cinco anos: revisão sistemática da literatura. Epidemiol. Serv. Saúde, Brasília,2017; 26(1): 169-182.

19. ROSSATO K. Acolhimento com classificação de risco na estratégia de saúde da família: percepção da equipe de enfermagem; UFSM; 2017;

20. SANTOS UP, et al. Poluição do ar ambiental: efeitos respiratórios J. bras. pneumol., São Paulo, 2021; 47(1): e20200267.

21. SILVEIRA A, NEVES ET. Crianças com necessidades especiais em saúde: cuidado familiar na preservação da vida. Ciência Cuidado e Saúde, 2016; 11(1): 74-80.

22. SOUZA RR, et al. A rede de atenção integral à saúde da criança no Distrito Federal, Brasil. Ciênc. saúde coletiva, Rio de Janeiro, 2019; 24(6): 2075-2084.

23. VIGANO HHG, et al. Incêndios no Pantanal de Corumbá, MS: modelagem e previsão a partir das técnicas de análise multivariada. Rev. Ambient. Água, Taubaté, 2018; 13(5): e2024. 